\title{
Erratum to: Genome co-amplification upregulates a mitotic gene network activity that predicts outcome and response to mitotic protein inhibitors in breast cancer
}

Zhi Hu ${ }^{1+}$, Jian-Hua Mao ${ }^{2+}$, Christina Curtis ${ }^{3+}$, Ge Huang ${ }^{1+}$, Shenda Gu', Laura Heiser ${ }^{1}$, Marc E. Lenburg ${ }^{4}$, James E. Korkola ${ }^{1}$, Nora Bayani², Shamith Samarajiwa ${ }^{5}$, Jose A. Seoane ${ }^{3}$, Mark A. Dane ${ }^{1}$, Amanda Esch', Heidi S. Feiler ${ }^{1}$, Nicholas J. Wang ${ }^{1}$, Mary Ann Hardwicke ${ }^{6}$, Sylvie Laquerre ${ }^{6}$, Jeff Jackson ${ }^{6}$, Kenneth W. Wood ${ }^{7}$, Barbara Weber ${ }^{6}$, Paul T. Spellman ${ }^{1}$, Samuel Aparicio ${ }^{8}$, Richard Wooster ${ }^{6}$, Carlos Caldas ${ }^{9^{*}}$ and Joe W. Gray ${ }^{1 *}$

\section{Erratum}

In the version of this article that was published on PubMed [1] the author's name "Mark A. Dane" was formatted incorrectly in the XML mark up and therefore appeared incorrectly on PubMed. In the XML mark up, the middle initial "A" was added as a Particle when it should have been included as a Given Name. Due to this error, the author's name was incorrectly formatted in PubMed as "A Dane M" and not as "Dane MA". The author's name is correct on the BioMed Central website. The author's name in the XML of the original article [1] has been updated accordingly.

\footnotetext{
Author details

'Department of Biomedical Engineering, School of Medicine, Oregon Health \& Science University, 3303 SW Bond Ave., CH13B, Portland, OR 97239, USA. 'Life Sciences Division, Lawrence Berkeley National Laboratory, Berkeley, CA 94127, USA. ${ }^{3}$ Department of Medicine, Division of Oncology and Department of Genetics, Stanford University School of Medicine, Stanford, CA 94305, USA. ${ }^{4}$ Department of Pathology and Laboratory Medicine, Boston University School of Medicine, Boston, MA 02215, USA. ${ }^{5}$ MRC Cancer Unit, University of Cambridge, Cambridge CB2 OXZ, UK. ${ }^{6}$ GlaxoSmithKline, Collegeville, PA 19425, USA. ${ }^{7}$ Cytokinetics, Inc., South San Francisco, CA 94080, USA. ${ }^{8}$ Molecular Oncology, BC Cancer Research Centre, Vancouver, Canada.

${ }^{9}$ Cancer Research UK, Cambridge Institute, Cambridge, UK.
}

Received: 1 February 2017 Accepted: 1 February 2017

Published online: 09 February 2017

\section{Reference \\ 1. Hu Z, Mao JH, Curtis C, Huang G, Gu S, Heiser L, et al. Genome co-amplification upregulates a mitotic gene network activity that predicts outcome and response to mitotic protein inhibitors in breast cancer. Breast Cancer Research. 2016;18:70. doi:10.1186/s13058-016-0728-y. \\ *Correspondence: carlos.caldas@cancer.org.uk; Grayjo@ohsu.edu \\ ${ }^{\dagger}$ Equal contributors \\ ${ }^{9}$ Cancer Research UK, Cambridge Institute, Cambridge, UK \\ 'Department of Biomedical Engineering, School of Medicine, Oregon Health \& Science University, 3303 SW Bond Ave., CH13B, Portland, OR 97239, USA}

